Quantum Mechanics of Particles and Wave Fields By Prof. Arthur March. Pp. x+292. (New York: John Wiley and Sons, Inc.; London : Chapman and Hall, Ltd., 1951.) 44s. net.

DROF. A. MARCH'S book is a very welcome addition to the literature of modern developments in the quantum theory of particles and wave fields. The changes in centres of interest in this subject are well indicated by the fact that one-half of this book is devoted to the subject of relativistic wave equations, and to the quantization of wave fields. The earlier chapters give a good introduction to the earlier developments in quantum theory, including, of course, Schrödinger's wave equation and the principles of matrix mechanics. The later chapters are peculiarly interesting and suggestive, as they deal with the central problems of quantum electrodynamics.

There is a curious passage on p. 179 which seems, at least on the surface, to be at variance with the usual interpretation of Dirac's wave equation. Prof. March writes: "It is important to realize the physical significance of the wave equation (248) [that is, Dirac's wave equation]. It has been emphasized already that this equation no Ionger permits an interpretation in the sense of non-relativistic quantum mechanics. As long as it is not quantized, it has no relation whatever with particles, but controls the behaviour of a field that is not purely symbolic but actually represents a physical reality. 'L'his is clear from the fact that, according to (249), (251), and (253), the field possesses charge, energy, and momentum, and thus it can be measured relative to these observables. It is only a question, then, as to whether wave fields corresponding to the assumed equation really exist, a question that must be answered in the affirmative". This interpretation is so unusual that Prof. March might well have referred to the fact that most writers, including Dirac himself, regard the relativistic wave equation as referring to the behaviour of an individual particle.

G. TEMple

The Lipids

Their Chemistry and Biochemistry. By Prof. Harry J. Deuel, Jr. Vol. 1 : Chemistry. Pp. xxiv +982. (New York and London: Interscience Publishers, Inc., 1951.) 18.50 dollars.

THE term lipid has been interpreted. in its fullest sense, and the author discusses not only the simple lipids (fats and waxes, 405 pages) but also the compound lipids (phosphatides and cerebrosides, 102 pages) and the derived lipids (carotenoids, 160 pages, and vitamins $A, 1), F$ and $K, 194$ pages). The author does not claim to be an authority in all these fields, though it is suggested, in the preface, that this will make the book less involved and therefore more understandable to non-specialist readers.

For so large a book, the number of mistakes is small, though some have been noted. For example, the formulæ on p. 168 (1:2-benzylidene glycerol), 428 (choline chloride) and 602 (azafrinone) are in error. It is stated (p. 88) that linolenic and elæostearic acids are the only octadecatrienoic acids known; but octadec-6:9:12-trienoic acid, reported to occur in Enothera biennis seed oil, is a member of this series. The statement (p. 242) concerning the low iodine value of linolenic acid seems doubtful, while the section on atmospheric oxidation of unsaturated fatty acids and their natural derivatives is somewhat inadequate, making no reference to the important new developments reported in this field by many workers during the past decade.

Despite these errors and omissions, the general style of the book is pleasing, and I have read it with profit and enjoyment. References are copious, and the indexes (12l pages) appear to be very complete. The book is most useful to the reader in those parts of the field in which he is not a specialist.

$$
\text { F. D. GUnstone }
$$

\section{A Basic Course in the Theory and Practice of Quantitative Chemical Analysis}

By Prof. George L. Clark, Prof. Leonard K. Nash and Prof. Robert B. Fischer. Pp. xxi+448. (Philadelphia and London: W. B. Saunders Co., 1949.) $21 s$.

UANTITATIVE analysis is one of the fundamental branches of the science of chemistry, and the proper instruction of the beginner in the broad essential aspects of the subject is invaluable to him in whichever field he may eventually specialize. rThis book, written by three authors with considerable experience in the teaching of analytical chemistry, forms an excellent introductory course for students.

Part 1 deals with gravimetric analysis, and Part 2 with volumetric analysis, the method of presentation in each case being a logical combination of theory and practice. Three representative determinations have been treated with unusual care and completeness in order to lead the inexperienced student to the proper critical approach to the subject, the later methods being dealt with in somewhat less detail. Part 3 describes briefly the modern instrumental methods of analysis. Many of the chapters are followed by review questions and problems which serve to test the reader's grasp of the subject-matter. Part 4 consists of ten short appendixes, including those on essential mathematics for quantitative analysis, standardization of weights and statistical treatment of experimental data.

The book is well written and well produced, but, from the British reader's point of view, the use of such terms as "precipitimetry" and "compleximetry" is to be deplored.

A Text-Book of Practical Organic Chemistry, including Qualitative Organic Analysis

By Dr. Arthur I. Vogel. Second edition. Pp. xxiii 1033. (London, New York and Toronto : Longmans, Green and Co., Ltd., 1951.) 50s. net.

THE issue of a second edition of this laboratory manual only three years after the publication of the first edition (see Nature, 161, 995; 1948) is evidence of the popularity which it has deservedly achieved. The new edition is, in fact, little more than a reprint. For the most part the pagination corresponds with that of the first edition; but it is stated that the opportunity has been taken to correct various minor errors and to incorporate some suggestions made to the author. The appendix is enlarged by the inclusion of examples of new preparative methods in common use. 'These are the formation of some acetylenic compounds by liquid ammonia reactions ; the use of $\mathrm{N}$-bromosuccinimide ; the Huang-Minlon modification of the WolffKishner reaction ; reductions with lithium aluminium hydride; cyanoethylation; and the use of organo lithium compounds. 'I'hese are welcome additions. There is no doubt that this book has established itself as a medium of instruction in practical organic chemistry. J. W. Cook 